\title{
Erythrocyte Mean Corpuscular Hemoglobin Concentration
}

National Cancer Institute

\section{Source}

National Cancer Institute. Erythrocyte Mean Corpuscular Hemoglobin Concentration.

NCl Thesaurus. Code C64798.

The mean cell hemoglobin concentration $(\mathrm{MCHC})$, which is the average concentration of hemoglobin in a given volume of blood. The MCHC is a calculated value derived from the measurement of hemoglobin and the hematocrit. 